EGU21-14229

https://doi.org/10.5194/egusphere-egu21-14229

EGU General Assembly 2021

(c) Author(s) 2021. This work is distributed under

the Creative Commons Attribution 4.0 License.

\title{
From Earth and beyond - Callio Lab underground centre for Science and R\&D
}

\author{
Julia Puputti, Jari Joutsenvaara, Ossi Kotavaara, and Eija-Riitta Niinikoski
}

University of Oulu, Kerttu Saalasti Institute, Nivala, Finland (hannah.puputti@oulu.fi)

Callio Lab is a unique research infrastructure operating at the Pyhäsalmi Mine in Finland [1]. It is coordinated by the University of Oulu Kerttu Saalasti Institute (KSI) [2]. Callio Lab is a key component of the larger CALLIO -Mine for Business concept aiming at repurposing the mine area into an economically feasible multidisciplinary operating environment [3]. Underground mining ends in autumn 2021 after which CALLIO continues to host activities at the mine-site until at least 2025.

Callio Lab has provided unique environments for fields of research ranging from physics and geosciences to underground food production and construction. As of spring 2021, Callio Lab has served as the host site for numerous international projects such as MINETRAIN, Goldeneye, and BSUIN. The MINETRAIN project developed intensive training courses for mining professionals through a holistic approach to the mine lifecycle [4]. Goldeneye is creating an artificial intelligence platform to improve safety, efficiency and profitability of mine sites in Europe [5]. BSUIN was headed by KSI and it piloted a method of thorough geophysical, structural, organizational and natural background radiation (NBR) characterization, making underground laboratories (UL's) more accessible for new and current users [6]. Opportunities in the fields of plant-based mineral exploration, geopolymers, circular economy, muography research as well as fire and blasting test sites are also being explored. Launched in 2018, the Callio SPACELAB initiative is dedicated to studies in space and planetary sciences. [1]

We have detailed knowledge of the overall geological structures, rock mechanics, and characteristics of the Callio Lab underground environments. This is due to the characterization activities performed during the BSUIN project, collected data materials from previous research, and the extensive microseismic network. Understanding the characteristics of the UL's is key in understanding the possibilities for R\&D and science. $[1,6]$

Callio Lab currently houses seven underground laboratories at different depths ranging from a shallow 75 meters underground all the way to the Main level and bottom of the mine at 1440 meters. There are two routes of access via the incline tunnel or the elevator shaft. The operating environment has good existing infrastructure and facilities, including electrical workshops, maintenance halls, a restaurant, offices, secure high-speed internet access, a logistically ideal single location, as well as the ability to provide innovation support and managing processes. Indepth understanding of underground risk management and the conditions of the working 
environment make Callio Lab a safe operating environment with vast opportunities and potential to grow into an internationally recognized research institute. [1]

[1] Callio Lab - Underground Center for Science and R \& D, www.calliolab.com, 8 Jan 2021

[2] Kerttu Saalasti Institute, www.oulu.fi/ksi-eng, 8 Jan 2021

[3] Mine for Business - Callio - Pyhäjärvi, Finland , www.callio.info, 8 Jan 2021

[4] MINETRAIN, www.minetrain.eu, 8 Jan 2021

[5] GoldenEye EU H2020 funded project, www.goldeneye-project.eu, 8 Jan 2021

[6] Baltic Sea Underground Innovation Network, www.bsuin.eu, 8 Jan 2021 